\title{
Acinetobacter baumannii Induced Pyogenic L1-L2 Spondylodiscitis
}

\author{
Swetabh Verma ${ }^{1}$, Vineesh Mathur ${ }^{2}$, Dharmendra Singh ${ }^{1}$, Mohammad Zahid Khan ${ }^{1}$ \\ ${ }^{1}$ Bone and Joints Institute, Medanta Lucknow; ${ }^{2}$ Bone and Joints Institute, Medanta Gurgoan, Haryana, India.
}

\section{Corresponding Author:}

Dr Swetabh Verma

Email: swetabhverma26@gmail.com

This is an Open Access article distributed under the terms of the Creative Commons Attribution License (creativecommons.org/ licenses/by/3.0).

Received

Accepted

Published
July 19, 2020

November 19, 2020

April 10, 2021

\begin{abstract}
Background: Axial back pain is primary presentation of infective spondylodiscitis due to which diagnosis is easily missed, especially in the absence of fever, or in patients admitted in intensive care unit with poor general condition. Prompt diagnosis established by biopsy helps to initiate guided antibiotics, thereby preventing further morbidity and mortality. Case Report: We present a rare such case of spondylodiscitis occurring at L1L2 disc space due to Acinetobacter baumannii, which was initially undiagnosed due to absence of clinically suggestive symptoms. An endoscopic disc space debridement, evacuation of pus, lavage and biopsy was performed subsequently. Conclusion: The case highlights the importance of having a strong suspicion of spondylodiscitis in susceptible patients, even in the setting of minimal clinical symptoms and the invaluable role of imaging techniques along with culture and biopsy to identify the pathology.
\end{abstract}

Keywords: Acinetobacter baumannii, Anti-Bacterial Agents, Back Pain, Biopsy, Debridement, Discitis, Endoscopy, Suppuration.

\section{Introduction}

Infection of spine can present with wide spectrum of clinical manifestations. Commonly, the intervertebral disc space, vertebral body, spinal canal and paraspinal structures are involved [1]. Based on etiology, the spinal infections can be classified as pyogenic, granulomatous (tubercular or fungal) or parasitic [2]. Spondylodiscitis represents $0.15 \%$ to $5 \%$ of all osteomyelitis cases [3]. There is a male predominance, with a male-to-female ratio of 1.5 to $2: 1$ [4]. Of recent, the incidence of infective spondylodiscitis has risen due to higher survival, immunocompromised states as HIV infection, intravenous drug abuse, healthcare-associated infection and better diagnostic modalities. Often at times, due to low index of clinical suspicion, there is a delay in diagnosis and patient presents with a neurological deficit. These are seen in about $30 \%$ of cases presenting with any neurological complication at diagnosis, and in about $60 \%$ of cases presenting with motor weakness [5]. We present a unique such of pyogenic spondylodiscitis wherein due to delayed diagnosis, the patient presented to us with loss of motor power in both lower limbs and significant axial pain. Prompt intervention by endoscopic disc space debridement, biopsy and copious lavage provided both a diagnostic and therapeutic advantage and patient made a significant recovery in the immediate post-operative period.

\section{Case Report}

A 48-year male, moderately built and nourished patient presented to our out-patient's clinic with complaints of axial back pain for two weeks and inability to move both lower limbs in the past five days. The axial back pain was dull aching in nature, non-radiating and aggravated on any movements. Patient also gave a history of being able to walk out of bed five days prior but since then he has developed gradual loss of power in both lower limbs. He does not report any history of fever, weight loss, intravenous drug abuse or 
chronic addictions. He had retained bowel and bladder functions. He was being treated at an elsewhere hospital conservatively but without significant relief of back pain. On examination, there was diffuse tenderness over dorsolumbar spine. Neurologically he had grade zero power in all myotomes below L1. There were no sensory dysfunctions. He had untreated bilateral congenital telipes equinovarus deformity and callosities on lateral border of feet due to chronic weight bearing [Fig.1]. He was a known case of type II diabetes mellitus on treatment for last ten years with a good glycemic control.

Plain radiographs of dorsolumbar spine showed decreased disc space at L1-L2 with end plates irregularity [Fig.2]. Magnetic resonance imaging showed hyper-intense signal changes within the L1-L2 disc space and marrow edema within the vertebral bodies. An epidural component was present at the same level with paravertebral collections with spinal cord compression and bilateral psoas edema. Note was also made of an arachnoidal cyst at D11-D12 level. There was contrast enhancement of the epidural component. Multiple prominent retroperitoneal lymph nodes were seen [Fig.3-5]. Rest of the spine screening was normal. The hematological parameters showed a high ESR and CRP level (120 mm at the end of first hour and $306 \mathrm{mg} / \mathrm{dL}$ respectively). The total leucocyte count was 15,800 cells $/ \mathrm{mm}^{3}$ with predominant polymorphs. Intravenous antibiotics were withheld till surgery.

The patient underwent endoscopic L1-L2 disc space debridement, biopsy and lavage under local anaesthesia and intravenous sedation the next day after admission. $5 \mathrm{~mL}$ of seropurulent fluid was aspirated from epidural space. Unhealthy tissue was debrided, disc space was irrigated with four to five litres of normal saline and epidural space was visualized by positioning the endoscope in halfand half view, which appeared healthy [Fig.6,7]. Following the procedure on day one of surgery, the patient had recovered motor power in both lower

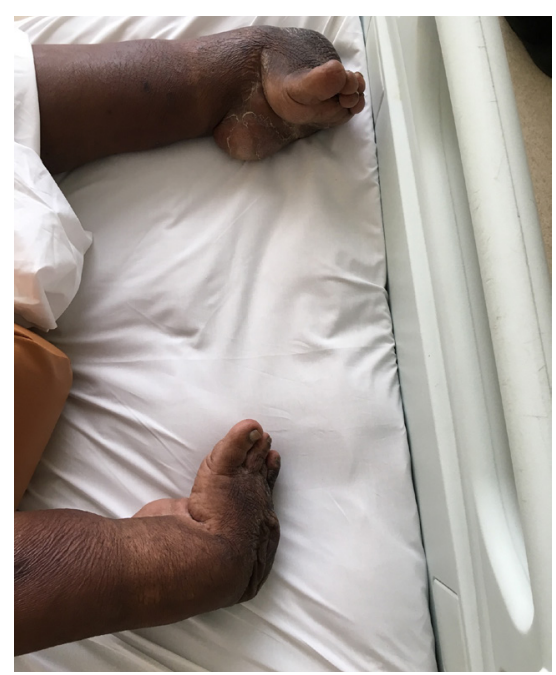

Fig.1: Bilateral foot deformity.

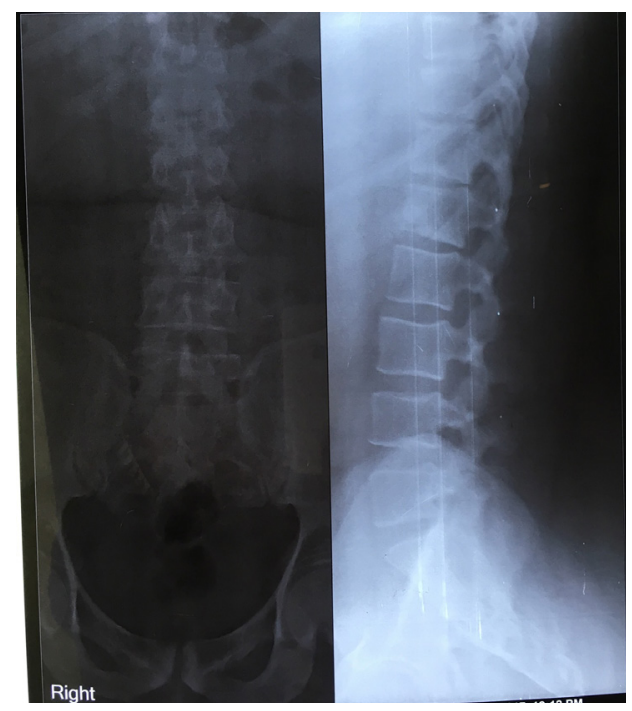

Fig.2: Plain radiograph of lumbosacral spine.

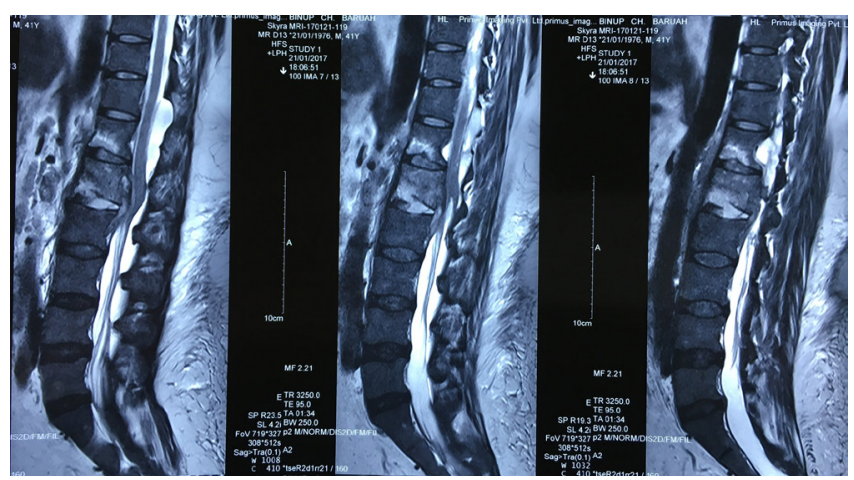

Fig.3: T2 sagittal. 
limbs from grade zero to grade three. His back pain also improved from VAS 8 to VAS 5 . He was made to sit at edge of bed and quadriceps exercises were initiated. The samples were negative for acid fast bacilli and fungus but multiple coccal forms were seen on gram stain. Gene-expert was negative for Mycobacterium tuberculosis. Tissue culture demonstrated the organism as Acinetobacter baumannii which was sensitive to colistin, clindamycin and gentamicin. The histopathology showed evidence of chronic osteomyelitis with no granulomas. The patient was started on culture directed antibiotics and by third post-operative day, he had significant relief of axial back pain. He was able to stand with support and walk a few steps. He was discharged on fourth day following surgery. Patient has been under follow up for last two years and is currently fully ambulatory [Fig.8].

\section{Discussion}

Spondylodiscitis is the infection of intervertebral disc and adjoining vertebrae, and one may predominate over the other [6]. It represents 3-5\% of all cases of osteomyelitis. At times, the term spondylitis is used to refer to a similar situation, which is basically osteomyelitis of the spinal column. Here the pathology starts at vertebral end plates, with secondary involvement of the intervertebral discs. Often times at presentation, inflammatory change are evident in both vertebral bodies and intervertebral disc, so that origin of the infection is not clear. Hence both the terms are commonly used. Primary infections occur directly within the spinal unit, secondary infections usually spread from a remote site. Postoperative discectomy patients developing discitis is sometimes seen with an incidence of $0.21 \%$ $3.6 \%$ [7]. Most common site of discitis is lumbar or thoracolumbar region [6]. Early diagnosis relies mostly on a combination of clinical, laboratory, and imaging findings.

With reference to symptomatology, fever is poorly sensitive to presence of spondylodiscitis

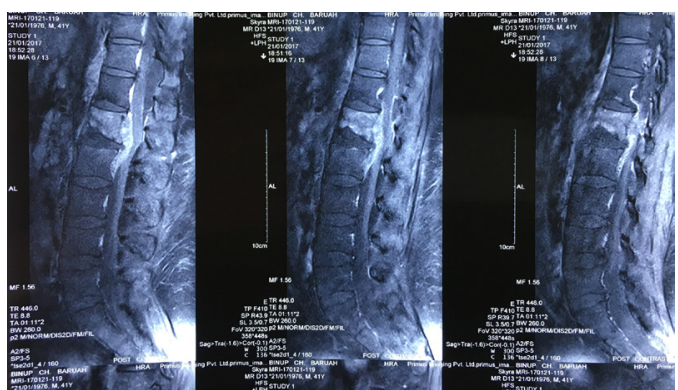

Fig.4: Contrast enhancement T1 section.

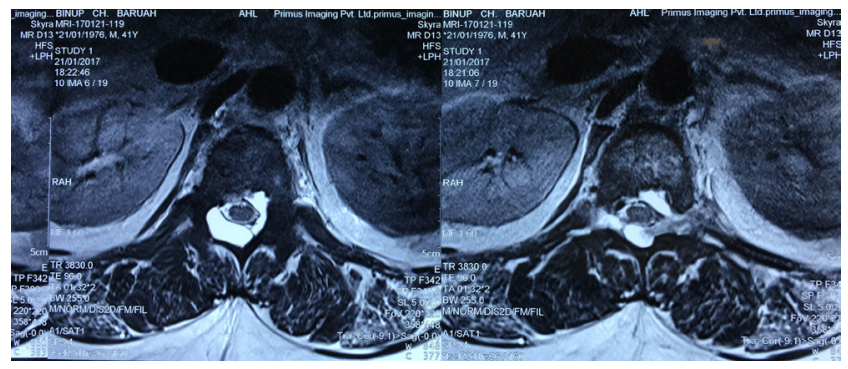

Fig.5: T2 axial image.

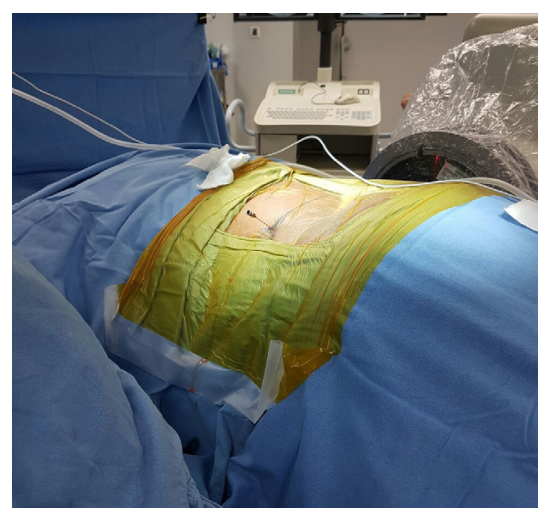

Fig.6: Puncture needle.

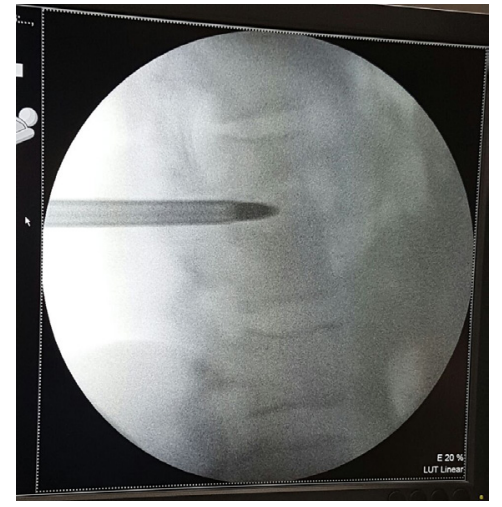

Fig.7: Channel (obturator) placement. 
with only $52-68 \%$ cases showing a febrile state [8-10]. On the other hand, back pain is the most common finding seen in more than $90 \%$ patients [11]. Studies report an incidence of neurological compression in $33-59 \%$ of patients. This is commonly apparent as radicular compression with consequent uni or bilateral weakness, parasthesia, or paralysis. Neurological symptoms indicate a mass effect from an abscess in the epidural space. The prevalence of abscess formation is varying, but cited in the literature as 35-74\% [12]. Another study by Kapeller et al. [10] showed that $60 \%$ presented with signs of radicular compression, and $30 \%$ with cord compression. Our patient had features of both spinal cord compression along with compression of roots of cauda equina.

The diagnosis is strongly suggested by a persistently elevated erythrocyte sedimentation rate (ESR), C-reactive protein (CRP), and by typical changes on magnetic resonance imaging (MRI). MRI with gadolinium scan is more sensitive. It is the radiographic imaging modality of choice in diagnosing pyogenic spondylodiscitis [13]. The total leucocyte counts are raised in only $35-60 \%$ cases [9]. It is remarkable to note that leukocytosis is more common in patients with MRI confirmed abscess formation, as seen in our case. The total leucocyte count was persistently high till the pathology was diagnosed. The CRP level was $>300 \mathrm{mg} / \mathrm{dL}$ at presentation. Raised ESR is seen in $>80 \%$ of patients with spondylodiscitis $[9,10,12]$. Most sources report the prevalence of positive blood cultures to be $55-75 \%$.

One of the important point of consideration is withholding the administration of antibiotics till biopsy samples are collected. Multiple studies have shown an increase in diagnostic yield with this step. Biopsies performed before initiation of antibiotics yield a causative organism in $75-80 \%$ of cases, while biopsies performed after initiation of antibiotics yield a causative microorganism in $50 \%$ cases [14]. Histology is highly sensitive in establishing the diagnosis of spondylodiscitis, but

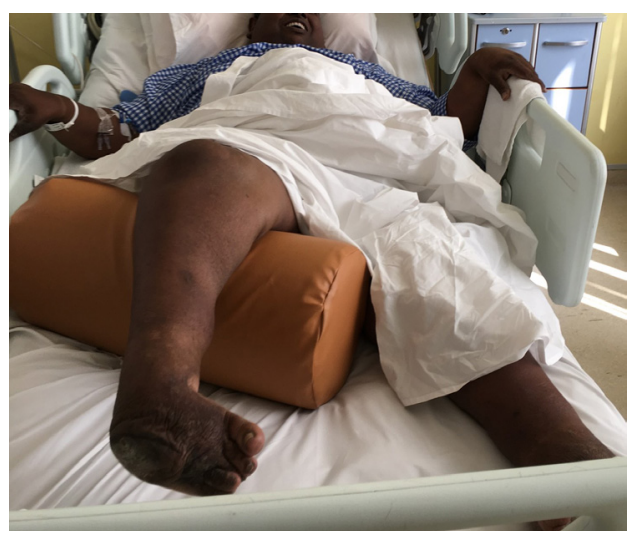

Fig.8: Post-operative recovery.

fails to identify the causative organism. Multiple studies report the sensitivity of histology between $81 \%$ and $90 \%$ and the specificity between $75 \%$ and $100 \%$ [15].

Percutaneous discectomy to diagnose and treat spondylodiscitis was reported in the 1990s. At present, abundant literature exists to support its use in medically compromised patients, where this procedure can be done under local anaesthesia. The success rate of biopsy has been reported from 86$100 \%$. Reduction of pain and final healing success ratio varies from $83 \%$ to $100 \%$ [16]. Even adnexal abscess, especially psoas abscess, can be drained [17]. We have specially found this technique useful in patients of spondylodiscitis in intensive care settings, because often times such patients are medically unfit for any procedure under general anaesthesia. Here this percutaneous endoscopic technique serves as an effective tool with high diagnostic yield as well as eliminating the foci of infection. At the same time, there is a direct visualisation of pathological tissue and neural decompression is ensured by proper positioning of endoscope.

Acinetobacter baumannii is a Gramnegative coccobacillus, has recently emerged as an important nosocomial pathogen. Its ability to acquire resistance to almost all groups of available antibiotics is a problem of great concern. Recent reports showed that most $A$. baumannii strains 
isolated in hospitals, especially in intensive care units (ICUs), are highly resistant to $\beta$-lactams, aminoglycosides, fluoroquinolones and carbapenems [18]. Colistin and rifampicin appears to be an effective and safe combination therapy for severe infections due to multidrug-resistant A. baumannii [19]. In our case, sensitivity was identified to colistin, which was started as soon as the culture result was available and the patient showed significant improvement in the back pain.

\section{Conclusion}

Pyogenic spondylodiscitis secondary to Acinetobacter baumannii must be kept as a differential diagnosis in susceptible patients even in the setting of absence of fever or elevated total leucocyte counts. A combination of serial monitoring of ESR and CRP levels, along with culture and histology serves as the most confirmative way to diagnose the condition. Administration of colistin is recommended based on drug sensitivity results. Percutaneous endoscopic disc space debridement and biopsy is a recent emerging and rapidly evolving minimally invasive technique especially appropriate in those medically unfit patients for any procedure under general anaesthesia.

Contributors: SV: manuscript preparation, proof reading and surgery; VM, DS: proof reading, and patient management; MZK: manuscript preparation and patient management. SV will act as guarantor. All authors approved the final version of this manuscript and are responsible for all aspects of this study.

Funding: None; Competing interests: None stated.

\section{References}

1. Jeong SJ, Choi SW, Youm JY, Hyun-Woo Kim, Ho-Gyun Ha, Jin-Seok Yi, et al. Microbiology and epidemiology of infectious spinal disease. J Korean Neurosurg Soc. 2014;56:21-27.

2. Kaufman DM, Kaplan JG, Litman N. Infectious agents in spinal epidural abscesses. Neurology. 1980;30:844850.

3. Sobottke R, Seifert H, Fätkenheuer G, Schmidt M, Gossmann A, Eysel P. Current diagnosis and treatment of spondylodiscitis. Dtsch Arztebl Int. 2008;105:181187.
4. Grammatico L, Baron S, Rusch E, Lepage B, Surer $\mathrm{N}$, Desenclos, JC, et al. Epidemiology of vertebral osteomyelitis (VO) in France: analysis of hospitaldischarge data 2002-2003. Epidemiol Infect. 2008;136:653-660.

5. Patel AR, Alton TB, Bransford RJ, Lee MJ, Bellabarba CB, Chapman JR. Spinal epidural abscesses: risk factors, medical versus surgical management, a retrospective review of 128 cases. Spine J. 2014;14:326-330.

6. Mulukutla RD. Postoperative spondylodiscitis. In: Rajasekaran S. Spinal Infections \& Trauma, New Delhi. Jaypee Brothers Medical Publishers. $1^{\text {st }}$ ed. 2011:307317.

7. Gerometta A, Bittan F, Olaverri JCR. Postoperative spondilodiscitis. International Orthopaedics (SICOT). 2012;36:433-438.

8. Perrone C, Saba J, Behloul Z, Salmon-Céron D, Leport C, Vildé JL, et al. Pyogenic and tuberculous spondylodiskitis (vertebral osteomyelitis) in 80 adult patients. Clinical Infectious Disease. 1994;19:746-750.

9. Hadjipavlou AG, Mader JT, Necessary JT, Muffoletto AJ. Hematogenous pyogenic spinal infections and their surgical management. Spine. 2002;25(13):1668-1679.

10. Kapeller P, Fazekas F, Krametter D, Koch M, Roob G, Schmidt R, et al. Pyogenic infectious spondylitis: clinical, laboratory and MRI features. European Neurology. 1997; 38(2):94-98.

11. Hopkinson N, Stevenson J, Benjamin S. A case ascertainment study of septic discitis: clinical, microbiological and radiological features. Quarterly Journal of Medicine. 2001;94:465-470.

12. Nolla JM, Ariza J, Gómez-Vaquero C, Fiter J, Bermejo $\mathrm{J}$, Valverde $\mathrm{J}$, et al. Spontaneous pyogenic vertebral osteomyelitis in nondrug users. Seminars in Arthritis and Rheumatism. 2002;31(4):271-278.

13. Maiuri F, Iaconetta G, Gallicchio B, Manto A, Briganti F. Spondylodiscitis. Clinical and magnetic resonance diagnosis. Spine (Phila Pa 1976). 1997;22:1741-1746.

14. Grados F, Lescure FX, Senneville E, Flipo RM, Schmit JL, Fardellone P. Suggestions for managing pyogenic (non-tuberculous) discitis in adults. Joint Bone Spine. 2007;74(2):133-139.

15. Michel SC, Pfirrmann CWA, Boos N, Hodler J. CTguided core biopsy of subchondral bone and intervertebral space in suspected spondylodiscitis. American Journal of Roentgeniology. 2006;185:977-980.

16. Fu TS, Yang SC, Tsai TT, Chen LH, Lai PL, Niu CC, et al. Percutaneous endoscopic debridement and drainage in immunocompromised patients with complicated infectious spondylitis. Minim Invasive Ther Allied Technol. 2010;19:42-47.

17. Choi EJ, Kim SY, Kim HG, Shon HS, Kim TK, Kim KH. Percutaneous endoscopic debridement and drainage with 
four different approach methods for the treatment of spinal infection; Pain Physician. 2017;20(6):E933-E940.

18. Gales AC, Jones RN, Forward KR, Liñares J, Sader HS, Verhoef J. Emerging importance of multidrugresistant Acinetobacter species and Stenotrophomonas maltophilia as pathogens in seriously ill patients: geographic patterns, epidemiological features, and trends in the Sentry Antimicrobial Surveillance Program [1997-1999]. Clin Infect Dis. 2001;32 Suppl 2:104-113. 19. Bassetti M, Repetto E, Righi E, Boni S, Diverio M, Molinari MP, et al. Colistin and rifampicin in the treatment of multidrug-resistant Acinetobacter baumannii infection. Journal of Antimicrobial Chemotherapy. 2008;61:417-420. 